\title{
A Distraction Can Impair or Enhance Motor Performance
}

\author{
Christopher Hemond, ${ }^{1}$ Rachel M. Brown, ${ }^{2}$ and Edwin M. Robertson ${ }^{1}$ \\ ${ }^{1}$ Center for Noninvasive Brain Stimulation, Harvard Medical School, Beth Israel Deaconess Medical Center, Boston, Massachusetts 02215, and ${ }^{2}$ Department \\ of Psychology, McGill University, Montreal, Quebec H3A 1B1, Canada
}

Humans have a prodigious capacity to perform multiple tasks simultaneously. Being distracted while, for example, performing a complex motor skill adds complexity to a task and thus leads to a performance impairment. Yet, it may not be just the presence or absence of a distraction that affects motor performance. Instead, the characteristics of the distraction may play a critical role in affecting human motor performance. Here, we show that performance of a motor sequence can be substantially enhanced by simultaneously learning an independent color sequence. In contrast, performance of the same motor sequence was impaired by concurrently counting the number of red cues that were in the color sequence. The color and motor sequences had different lengths (10 vs 12 items), different numbers of elements (five vs four elements), and different temporal patterns (randomly intermittent vs continuous) and thus were independent of one another. These observations show that distracting information does not always impair motor performance, and so is not a sufficient explanation for the impaired performance. Instead, the influence that a distraction exerts upon performance is mediated by the type of processes engaged: when similar core processes are engaged, motor performance is enhanced, whereas when very different processes are engaged (i.e., counting and sequence performance), performance is impaired. Thus, these observations deepen our understanding of how a distraction, depending on its characteristics, can either impair or enhance performance and may offer novel approaches to optimizing human cognition.

\section{Introduction}

We have all seen them, and perhaps feared them: individuals driving with one hand on the steering wheel while they talk on the phone. Our fear is perhaps justified, because being distracted when performing complex motor tasks, such as driving a car, can have devastating and potentially life-threatening effects upon our performance (Redelmeier and Tibshirani, 1997). Reflecting such everyday experience are studies showing that adding a distractive element to a motor task can impair performance. For example, performing a motor sequence can be impaired when participants simultaneously discriminate between the numbers of interleaved high- and low-pitched tones (Cohen et al., 1990; Rah et al., 2000; Keele et al., 2003). According to theory, adding an additional distractive element to a task impairs performance either because the complexity of the task has increased or because the number of tasks being performed has increased (Norman and Bobrow, 1975; Schmidtke and Heuer, 1997; Rah et al., 2000). In these theories, the presence of a distraction impairs motor performance. Yet, it may not be just the presence or absence of a distraction that affects motor performance; instead, the distraction's characteristics may critically affect performance.

Received Sept. 11, 2009; revised Nov. 4, 2009; accepted Nov. 11, 2009.

Financial support was provided by National Institutes of Health Grants R01 NS051446 and NS051446-03S1 (E.M.R.) and the National Science Foundation, Division of Behavioral and Cognitive Sciences Grant 0921177 (E.M.R.) We are grateful to Albert Galaburda and Chris Miall for their constructive thoughts and comments on this work.

Correspondence should be addressed to Edwin M. Robertson, Center for Noninvasive Brain Stimulation, Harvard Medical School, Beth Israel Deaconess Medical Center, 330 Brookline Avenue, Kirstein Building KS-221, Boston, MA 02215. E-mail: emrobert@bidmc.harvard.edu.

DOI:10.1523/JNEUROSCI.4592-09.2010

Copyright $\odot 2010$ the authors $\quad 0270-6474 / 10 / 300650-05 \$ 15.00 / 0$
Motor performance may be impaired when the distraction and the rest of the task are very different (sequence performance vs tone discrimination), as has been observed in earlier work, whereas when similar processes are engaged (sequence performance vs sequence learning), motor performance may be enhanced (Cohen et al., 1990; Rah et al., 2000; Keele et al., 2003). For example, performance on a sequence of movements may be enhanced by learning to accurately recall another independent sequence of items. Alternatively, engaging similar processes may cause a competition for limited neuronal resources and so increase the motor performance impairment associated with a distraction. In sum, there are three broad possibilities. Motor performance may be little changed by a distraction's characteristics and, consistent with contemporary theories, all that may matter is the presence or absence of the distraction. A second possibility is that changing the distracter's characteristics may intensify the performance impairment. A third possibility is that changing the distracter's characteristics may cause motor performance to be enhanced. We sought to distinguish among these possibilities by contrasting performance across four different tasks. In one task each element was similar, requiring the performance of a motor sequence plus the acquisition of an independent and distinct color sequence (sequence task); in another task the distraction and the rest of the task were different, requiring the performance of a motor sequence plus the counting of colored stimuli (counting task). We also used a task in which the order of the color cues was random, but participants were told that there was a color sequence (random task). In the final task there was no distraction, and participants performed only the motor sequence (standard task). Contrasting across these tasks allowed us to explore whether performance of a motor sequence 


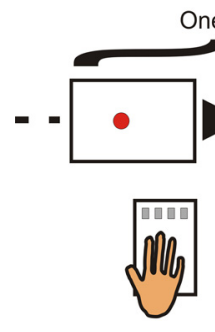

Visual cue

A ne trial
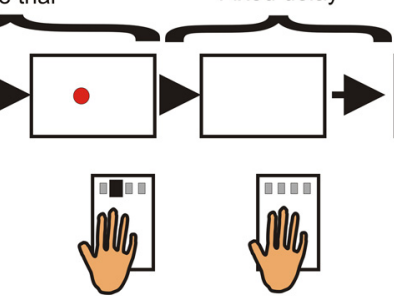

Response selected

Sequence task
B

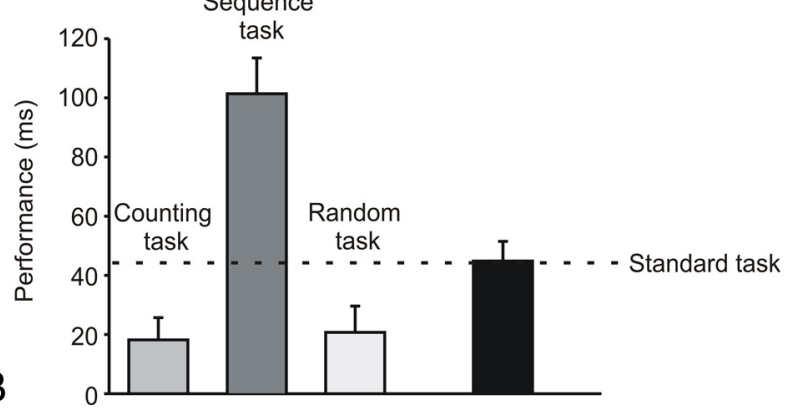

Figure 1. A, Participants saw a visual cue appear and responded by selecting the appropriate response button, as indicated by the position of the cue. Having selected the appropriate response button the cue disappeared, ending the trial, and after a fixed delay, another visual cue appeared marking the beginning of a new trial. The position and the color of the visual cues played out two independent sequences. All participants responded to the position of the visual cue, guiding the performance of a motor sequence and, in three groups, participants also simultaneously performed another element of the task. $\boldsymbol{B}$, Motor sequence performance was impaired by simultaneously counting the number of red colored visual cues (counting task; bar plot, mean \pm SEM). In contrast, performance was enhanced when participants performed one sequence guided by the position of the visual cue while simultaneously learning another independent sequence played out by the color of the visual cue (sequence task). Knowing of the color sequence was not sufficient to cause enhanced motor performance, because there was impaired sequence performance even when participants were told of the color sequence, which, in fact, had been replaced with a series of pseudorandom color changes (random task). These three tasks were contrasted against motor sequence performance in a task that only required participants to perform a motor sequence guided by the position of visual cue (standard task).

can be influenced not only by the presence or absence of a distraction but also by the similarity between task elements.

\section{Materials and Methods}

Participants. Forty-eight right-handed (defined by the Edinburgh handedness questionnaire) (Oldfield, 1971) participants (22 male, $20.1 \pm 2.1$ years) were randomly and equally distributed among four groups.

Experimental design. Two independent sequences were played out within a modified version of the serial reaction time task (see below, Serial reaction time task) (Nissen and Bullemer, 1987; Robertson, 2007) (Fig. 1A). One sequence was represented by color, whereas the other was represented by the position of the visual cue. In one group, participants were told that the color of the visual cue played out a sequence and were instructed to search for this sequence (sequence task). In another group, participants were not told of the color sequence and were instructed to count the total number of red visual cues (counting task). Thus, in the sequence task the distraction (color sequence) and performance (motor sequence) called upon similar processes (i.e., arranging events in a temporal order), whereas in the counting task the distraction (color counting) did not require any temporal order to be generated and so was very different from the processes required for performance (motor sequence). There were also two control groups. The first was used to understand the importance of the instruction to learn a color sequence. In this group there was no color sequence and instead the color of the cue was random; nonetheless, participants were told that there was a color sequence and instructed to search for this sequence (random task). Finally, to provide a measure of performance without the influence of instruction or distraction we used a task in which the distractive element was removed (standard task). Across all these groups, participants were introduced to the task as a test of reaction time and instructed to respond to the position of the visual cue.

Serial reaction time task. We used a modified version of the serial reaction time task (Nissen and Bullemer, 1987; Robertson, 2007). In this task a circular visual cue, filled with one of five colors [red ( $r$ ), yellow $(y)$, green (g), blue (b), or purple (p)], appeared at any one of four positions arranged horizontally on a computer screen. Each screen position, designated $1-4$, corresponded to a button on a response pad. When the cue appeared at the start of each trial, a participant selected the appropriate response button to end the trial (Fig. $1 A$ ). After a short fixed delay of 400 $\mathrm{ms}$ another colored cue occupying a new position was presented. The color of the visual cue played out a 10-item sequence (r-p-y-r-g-p-b-y$\mathrm{g}-\mathrm{b})$, while the position of the visual cue independently played out a distinct 12-item sequence (2-3-1-4-3-2-4-1-3-4-2-1). Each of these different sequences was differentially related to a set of random trials. For the color sequence, there were short blocks randomly containing two to four repetitions of the sequence (i.e., 20-40 trials), which were interleaved with a random number of trials $(1-3)$ of random color. In contrast, for the position sequence there was a single block of 180 trials of the 12 -item sequence preceded and followed by 50 random trials. Thus, there was no consistent relationship between the color and position sequences, allowing them to be played out independently. Within the random trials there were no item repeats and each set of random trials was unique. However, the random trials were identical across all groups. At completion, a free recall test for the position and color sequences was administered.

Data analysis. Having appeared at a particular position, the visual cue remained until the appropriate response button was selected. The duration between the appearance and the disappearance of the cue provided participants' response time. Any response time $>2.7$ SDs (i.e., the top 1st percentile) from a participant's mean was removed, as was any response time exceeding $3000 \mathrm{~ms}$. A sensitive and specific measure of task performance measure can be obtained by contrasting the sequential response times against those response times for the random trials (Nissen and Bullemer, 1987; Willingham et al., 1989; Robertson, 2007). Here, performance upon the position sequence was calculated by subtracting the average response time of the final 50 random trials from the average response time of the preceding 50 sequential trials (Nissen and Bullemer, 1987; Willingham et al., 1989). An ANOVA was used to compare performance among the groups and unpaired $t$ tests were used to compare the performance of the position sequence between groups.

\section{Results}

As a measure of performance across the three tasks, we calculated the difference in response time between the sequential and the subsequent random position trials, which provides a widely used, sensitive, and specific measure of motor sequence performance (Nissen and Bullemer, 1987; Robertson, 2007). We found that across the four tasks there was a significant difference in motor sequence performance (ANOVA; $F_{(3,44)}=14.5, p<0.001$ ) (Fig. $1 B)$. Performance was impaired when participants counted the number of red stimuli compared with when they only responded to the stimulus position $(18 \pm 8$ vs $44 \pm 7 \mathrm{~ms}$, mean $\pm \mathrm{SEM}$, counting task vs standard task; unpaired $t$ test, $t_{(22)}=2.65, p=$ 0.014 (Fig. $1 B$ ). These results, consistent with previous studies, show that adding a distracter element, in this case asking participants to count the number of red stimuli, can impair motor sequence performance (Cohen et al., 1990; Schmidtke and Heuer, 1997; Rah et al., 2000). In contrast, when participants learned the color sequence motor performance was greater than when participants either counted the number of red stimuli (98 \pm 14 vs $18 \pm 8 \mathrm{~ms}$, sequence task vs counting task; unpaired $t$ test, $\left.t_{(22)}=4.992, p<0.001\right)$ or simply responded to the posi- 


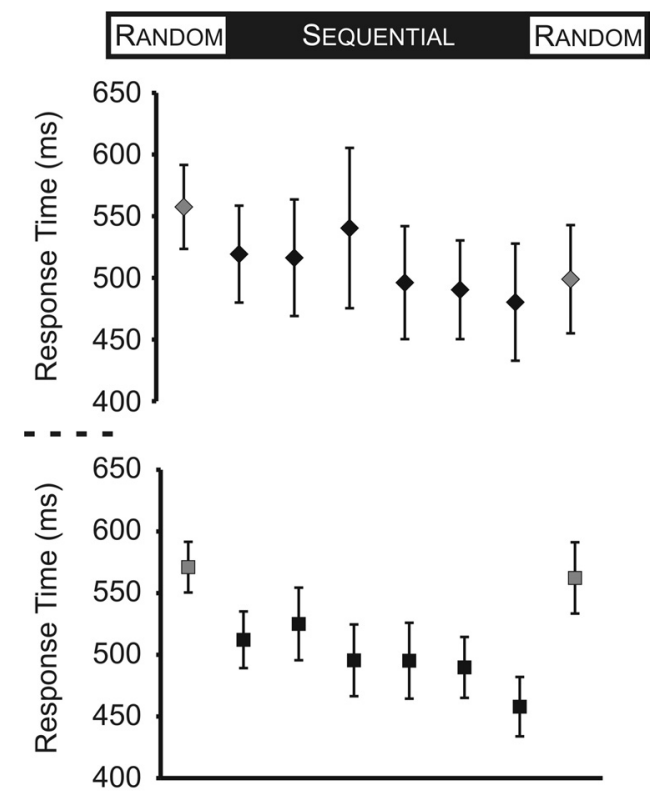

Trials

Figure 2. Participants' response time to the initial random trials (gray; mean $\pm S E M$ ) did not differ significantly between those counting the number of red visual cues (counting task, diamonds) and those learning the sequence of colors played out by the visual cue (sequence task, squares; unpaired $t$ test, $t_{(22)}=0.340, p=0.737$ ). Similarly, response times to the sequential trials did not differ significantly between the groups (counting task vs sequence task; unpaired $t$ test, $t_{(22)}=0.339, p=0.738$ ). However, replacing the position sequence with random trials caused a greater increase in response time in those learning the independent color sequence than in those counting the number of red visual cues (unpaired $t$ test, $t_{(22)}=$ $4.992, p<0.001)$. The greater increase in response time when the sequential trials were replaced by random trials indicates that those learning the independent color sequence had come to anticipate and expect the repeating position sequence to a greater extent than those counting the number of red visual cues (Willingham et al., 1989; Robertson, 2007). Thus, learning a color sequence enhanced participants' performance of an independent position sequence.

tion of the visual stimuli ( $98 \pm 14$ vs $44 \pm 7 \mathrm{~ms}$, sequence task vs standard task; unpaired $t$ test, $t_{(22)}=3.41, p=0.003$ ) (Fig. $1 B$ ). Recall for the color sequence was significantly greater when participants were asked to learn the color sequence as opposed to counting the number of red cues $(5 \pm 0.61$ vs 0 items, sequence task vs counting task; unpaired $t$ test, $t_{(22)}=8.1, p<0.001$ ). Thus, learning a color sequence did not impair motor performance, as occurred during concurrent counting, but instead enhanced performance of the independent motor sequence.

We sought to determine whether differences between the two experimental tasks (sequence vs counting) had produced a general improvement in task performance by examining individual response times. For example, the properties of one task may have promoted faster response times to a visual cue. Participants' response times to the initial random trials, which preceded the sequential trials, did not differ significantly between those concurrently learning a color sequence compared with those counting the number of red cues (sequence task vs counting task; unpaired $t$ test, $\left.t_{(22)}=0.340, p=0.737\right)$. During the subsequent sequential trials these groups showed a significant decrease in response time (sequence task and counting task; ANOVA, $F_{(5,110)}=$ 2.48, $p=0.035$ ) (Fig. 2). Participants' response time during the sequential trials did not differ significantly between the groups (sequence task vs counting task, $472 \pm 20$ vs $490 \pm 45$; unpaired $t$ test, $\left.t_{(22)}=0.339, p=0.738\right)$. Thus, despite the different properties of each task there was not a general difference in task performance. However, when the sequential trials were replaced with random trials there was a greater increase in response time for those learning the independent color sequence than for those counting the number of red visual cues (unpaired $t$ test, $t_{(22)}=$ $4.992, p<0.001$ ) (Fig. 2). The greater increase in response time, when the sequential trials were replaced by random trials, indicates that those learning the independent color sequence had come to anticipate and expect the repeating position sequence to a far greater extent than those counting the number of red visual cues (Willingham et al., 1989; Robertson, 2007). Our observations suggest that there was little or no general improvement in task performance from concurrently learning a color sequence in comparison with counting colored cues; instead, there was an enhancement specifically of motor sequence performance.

Knowing that a color sequence was present may have encouraged participants to seek other sequences, leading to the discovery of the position sequence and, in turn, to the enhanced performance of that sequence. However, those who knew of the color sequence (sequence task) and so may have been prompted to seek other sequences were not able to describe significantly more of the position sequence than those who had not known (counting task) of the color sequence ( $1 \pm 0.5$ vs $0.8 \pm 0.5$ items, sequence task vs counting task; unpaired $t$ test, $t_{(22)}=0.56, p=$ $0.58)$. Thus, knowing that there was a color sequence did not increase participants' knowledge of the motor sequence and so indirectly enhance motor performance.

Simply instructing participants to search for a color sequence may have been sufficient to enhance motor performance. In a separate group of subjects, we examined this possibility by instructing participants to search for a color sequence when in fact the color stimuli were randomly presented. In this group, participants' performance was substantially less than when the color stimuli followed a sequence $(21 \pm 9$ vs $98 \pm 14 \mathrm{~ms}$; unpaired $t$ test, $\left.t_{(22)}=4.64, p<0.001\right)$ and was no greater than when participants had counted the number of red stimuli ( $21 \pm 9$ vs $18 \pm$ $8 \mathrm{~ms} ; t_{(22)}=0.220, p=0.828$ (Fig. $1 B$ ). Thus, merely knowing of the color sequence was not sufficient to enhance motor performance; instead, having an independent color sequence to learn was vital for enhanced performance. Recall of the position sequence did not differ significantly between the groups in which the color cues were random compared with those in which the color cues followed a sequence $\left(1.1 \pm 0.5\right.$ vs $0.4 \pm 0.3 ; t_{(22)}=1.3$, $p=0.184)$, implying that the color sequence did not make the position sequence easier to identify and therefore easier to perform.

\section{Discussion}

Here, we show that the motor performance of a sequence can be substantially enhanced by concurrently learning an independent sequence of colored cues. In contrast, performance of the same motor sequence was impaired by concurrently counting the number of red cues that were in the color sequence. So, adding distracting information is not sufficient to impair performance. Instead, it is the particular processes engaged, rather than the amount or complexity of information being processed, which determines how a distraction affects motor performance. When similar processes are engaged, such as learning a color sequence and an independent motor sequence, then motor performance is enhanced; whereas, when distinct processes are engaged, such as counting and sequence performance, then motor performance is impaired. These observations provide a new perspective upon the mechanism through which distraction affects motor performance; rather than the amount or complexity of information 
determining performance, it is the processes engaged that determines performance.

A number of mechanisms could explain how concurrent color sequence learning enhanced motor sequence performance. One broad possibility is that the sequence of cue positions that guides motor sequence performance is made more obvious by the color sequence. There are a number of ways in which this could be achieved. For example, having a color and motor sequence of the same length and having each sequence maintain a consistent relationship with the other throughout the task would enhance motor sequence performance. An extreme example of this is for both sequences to be 10 items long and each repetition of each sequence to start and end together; in effect, the color and position cues would play out the same sequence. This arrangement of having a color and a position sequence coupled together can substantially enhance motor sequence performance (Robertson and Pascual-Leone, 2001). However, in the current work the color and position sequences were not coupled to one another; instead, each sequence was independent of the other. The position sequence was 12 items in length, consisting of four different positions and presented as a single block containing multiple iterations of the sequence; in contrast, the color sequence was 10 items in length, consisting of five different colors and presented as multiple short iterations of the sequence interleaved among random trials. Such a design ensured that the color and position sequences remained independent of one another, and so it was not possible for the color sequence to enhance motor performance by making the position sequence more salient. Recall of the position sequence was not significantly different when the color cues followed a sequence as opposed to being random, which demonstrated that the color sequence did not make the position sequence more salient. Finding that the color sequence enhanced motor sequence performance but did not enhance recall of the sequence implies that the acquisition of motor skill may occur independently from the acquisition of knowledge for the sequence. Similarly, other studies have shown that motor skill and knowledge of a sequence can be acquired in parallel and independently (Willingham and Goedert-Eschmann, 1999; Willingham et al., 2002). Thus, we used a design that ensured the color and position sequences remained independent; consistent with this independence, the color sequence did not improve recall of the position sequence, and so it seems unlikely that the color sequence was able to enhance motor performance by making the position sequence more salient.

The distractive element of a task, such as distinguishing between high- and low-pitched tones, can become integrated with the performance of a motor sequence (Schmidtke and Heuer, 1997; Rah et al., 2000; Keele et al., 2003). As a consequence, participants no longer perform two distinct tasks; instead, they are performing a single integrated task. Integration between the distractive element and the motor component has been associated with a decrease in motor performance. So, integration between task elements alone does not provide a sufficient explanation for concurrent color sequence learning enhancing motor performance.

Learning a color sequence may prompt participants to seek other sequences and so lead to enhanced performance of the motor sequence. Yet, participants' ability to recall segments of the position sequence was no greater when concurrently learning a color sequence as opposed to counting colored cues. Knowing that a color sequence was present did not enhance participants' ability to recall the position sequence, perhaps because humans invariably search and expect to find order among a series of events regardless of the instructions given to them (Wolford et al., 2004). As a consequence, participants were probably searching for sequences among the trials whether or not they were instructed to do so, and so their recall for the position sequence was not affected by the instruction to search for a color sequence. Thus, the instruction to search for a color sequence does not appear to increase participants' propensity to search for other sequences, and so enhanced searching is unlikely to account for enhanced motor sequence performance. Nonetheless, the instruction to search for a color sequence may directly enhance motor performance without having a detectable effect upon recall of the position sequence. However, when participants are instructed to search for a color sequence when in fact the color stimuli were randomly presented, there was no enhancement of motor performance. Thus, the instruction to search for a color sequence alone is not sufficient to enhance motor performance.

Color sequence learning and color counting are both distractions from performing a motor task; however, only color counting leads to impaired performance of a motor sequence (Norman and Bobrow, 1975; Keele et al., 2003). The nature of the distraction (i.e., sequence learning vs counting) and not just the presence or absence of the distraction helps to determine motor sequence performance. Specifically, concurrent color counting impairs motor performance, whereas concurrent learning of an independent color sequence enhances motor performance (Fig. 1). The capacity for different distractions to have distinct effects upon performance may be because the overlap or similarity of processing between the distraction and the motor task determines performance. For example, a common feature of color sequence learning and motor sequence performance is the generation of a sequence. These common features may engage a common process responsible for establishing the temporal order of a series of events (i.e., a sequence) regardless of how those events are expressed as a sequence (movements vs colors). Alternatively, acquiring a motor sequence and an independent color sequence are both learning processes, and consequently, it may be the simultaneous engagement of learning processes that produces enhanced motor performance. Yet, enhanced performance can occur in word-naming tasks, which lack a learning component; for example, enhanced performance occurs when a word is simultaneously presented along with colors, pictures, and related words that engage common processes (Rayner and Posnansky, 1978; Duncan-Johnson and Kopell, 1981; MacLeod, 1991; Mahon et al., 2007). Moreover, adapting reaching movements to an external force field does not enhance the simultaneous acquisition of an independent motor sequence (Overduin et al., 2008). Thus, having several features of a task simultaneously engage learning mechanisms is neither necessary nor sufficient to explain enhanced task performance. Nonetheless, having learning as a common feature along with sequencing may have added to the enhanced performance, making it greater than that achieved with only sequencing as a common component. Similarly, increasing the number of sequences within the task may increase the performance enhancement still further, although it seems unlikely that this would be a simple linear relationship.

Multiple independent sequences within a task may engage a common neural circuit. A network that includes areas of the prefrontal and parietal cortices has been implicated in supporting the representation of sequences, regardless of how those sequences are performed (Grafton et al., 1998; Hikosaka et al., 2002; Robertson, 2009). Activating a circuit, such as one devoted to sequence processing, may be facilitated when that circuit has already been engaged, for example, by a task involving sequenc- 
ing (Silvanto et al., 2008; Pasley et al., 2009). So, having multiple simultaneous demands for sequential processing may make it easier to engage the sequencing capacity of the brain and so in turn enhance performance of the motor sequence. In contrast, performance is impaired when processes that have little in common are engaged, for example, color counting and simultaneously performing a sequence.

The engagement of different processes may also explain the interference that occurs when two sequences are acquired in quick succession (Walker et al., 2003). When one motor sequence (memory A) and then another (memory B) are acquired in quick succession, the second of the two memories (memory B) is retained, but recall for the earlier memory (i.e., memory A) is impaired. The standard explanation for this interference is that there are insufficient neuronal resources to support the processing of both memories in quick succession (Brashers-Krug et al., 1996; Walker et al., 2003; Robertson et al., 2004). Instead, the interference may arise because two distinct processes are being engaged. The initial motor sequence is being consolidated by off-line processes (i.e., memory A), while encoding processes are being engaged to support the acquisition of the latter sequence (i.e., memory B). Distinct memory processes may compete with one another and so produce impaired performance (Huijbers et al., 2009). Thus, the principle that performance is impaired when distinct processes are engaged may not only serve to explain our current observations but also provide a framework for understanding the interference between memories.

A distraction, rather than impairing motor sequence performance by increasing the number of tasks or complexity of the task, instead may impair performance by causing multiple, distinct processes with little in common to be engaged. Conversely, motor performance can be enhanced when similar processes are engaged; for example, learning one sequence enhances the concurrent performance of an independent motor sequence. These observations suggest that it is not the amount or complexity of information within a task that limits human performance; instead, it is the simultaneous engagement of distinct processes that limits human performance.

\section{References}

Brashers-Krug T, Shadmehr R, Bizzi E (1996) Consolidation in human motor memory. Nature 382:252-255.

Cohen A, Ivry R, Keele SW (1990) Attention and structure in sequence learning. J Exp Psychol Learn Mem Cogn 16:17-30.

Duncan-Johnson CC, Kopell BS (1981) The Stroop effect: brain potentials localize the source of interference. Science 214:938-940.

Grafton ST, Hazeltine E, Ivry RB (1998) Abstract and effector-specific representations of motor sequences identified with PET. J Neurosci 18:9420-9428.
Hikosaka O, Nakamura K, Sakai K, Nakahara H (2002) Central mechanisms of motor skill learning. Curr Opin Neurobiol 12:217-222.

Huijbers W, Pennartz CM, Cabeza R, Daselaar SM (2009) When learning and remembering compete: a functional MRI study. PLoS Biol 7:e11.

Keele SW, Ivry R, Mayr U, Hazeltine E, Heuer H (2003) The cognitive and neural architecture of sequence representation. Psychol Rev 110:316-339.

MacLeod CM (1991) Half a century of research on the Stroop effect: an integrative review. Psychol Bull 109:163-203.

Mahon BZ, Costa A, Peterson R, Vargas KA, Caramazza A (2007) Lexical selection is not by competition: a reinterpretation of semantic interference and facilitation effects in the picture-word interference paradigm. J Exp Psychol Learn Mem Cogn 33:503-535.

Nissen MJ, Bullemer P (1987) Attentional requirements of learning: evidence from performance measures. Cogn Psychol 19:1-32.

Norman DA, Bobrow DG (1975) On data-limited and resource limited processes. Cogn Psychol 7:44-64.

Oldfield RC (1971) The assessment and analysis of handedness: the Edinburgh inventory. Neuropsychologia 9:97-113.

Overduin SA, Richardson AG, Bizzi E, Press DZ (2008) Simultaneous sensorimotor adaptation and sequence learning. Exp Brain Res 184:451-456.

Pasley BN, Allen EA, Freeman RD (2009) State-dependent variability of neuronal responses to transcranial magnetic stimulation of the visual cortex. Neuron 62:291-303.

Rah SK, Reber AS, Hsiao AT (2000) Another wrinkle on the dual-task SRT experiment: it's probably not dual task. Psychon Bull Rev 7:309-313.

Rayner K, Posnansky C (1978) Stages of processing in word identification. J Exp Psychol Gen 107:64-80.

Redelmeier DA, Tibshirani RJ (1997) Association between cellular-telephone calls and motor vehicle collisions N Engl J Med 336:453-458.

Robertson EM (2007) The serial reaction time task: implicit motor skill learning? J Neurosci 27:10073-10075.

Robertson EM (2009) From creation to consolidation: a novel framework for memory processing. PLoS Biol 7:e19.

Robertson EM, Pascual-Leone A (2001) Aspects of sensory guidance in sequence learning. Exp Brain Res 137:336-345.

Robertson EM, Pascual-Leone A, Miall RC (2004) Current concepts in procedural consolidation. Nat Rev Neurosci 5:576-582.

Schmidtke V, Heuer H (1997) Task integration as a factor in secondary-task effects on sequence learning. Psychol Res 60:53-71.

Silvanto J, Muggleton N, Walsh V (2008) State-dependency in brain stimulation studies of perception and cognition. Trends Cogn Sci 12:447-454.

Walker MP, Brakefield T, Hobson JA, Stickgold R (2003) Dissociable stages of human memory consolidation and reconsolidation. Nature 425:616-620.

Willingham DB, Goedert-Eschmann K (1999) The relation between implicit and explicit learning: evidence for parallel development. Psychol Sci 10:531-534

Willingham DB, Nissen MJ, Bullemer P (1989) On the development of procedural knowledge. J Exp Psychol Learn Mem Cogn 15:1047-1060.

Willingham DB, Salidis J, Gabrieli JD (2002) Direct comparison of neural systems mediating conscious and unconscious skill learning. J Neurophysiol 88:1451-1460.

Wolford G, Newman SE, Miller MB, Wig GS (2004) Searching for patterns in random sequences. Can J Exp Psychol 58:221-228. 